\title{
Spatial Heterogeneity and Factors Influencing Anemia and Severe Anemia among Under-5 Children in Ethiopia: Spatial and Multilevel Analysis
}

Bayuh Asmamaw Hailu ( $\sim$ bayuhasmamaw@gmail.com )

Wollo University

Getahun Gebre Bogale

Wollo University

Birhan Asmame Miheretu

Wollo University

Bisrat Misiganaw Geremew

University of Gondar

Joseph Beyene

McMaster University Department of Clinical Epidemiology and Biostatistics

Research article

Keywords: Child, Anemia, Sever anemia, Multilevel, Spatial

Posted Date: May 11th, 2020

DOI: https://doi.org/10.21203/rs.3.rs-25582/v1

License: (c) (i) This work is licensed under a Creative Commons Attribution 4.0 International License. Read Full License 


\section{Abstract}

Background: Anemia is recognized as a significant public health problem in Ethiopia.

Method: This secondary analysis, sampling technique and procedures done by DHS. A total of 8482 children aged 6-59 months were included in the study from EDHS 2016. Used tools for spatial heterogeneity analysis are Morans I, Local G*, Heat map, and Kulldorff's scan statistic, those carried out open source software (QGIS, GeoDa, SaTScan). Multilevel logistic regression analysis was used to identify both individual and household level factors associated with anemia and severe anemia, which measures between household variability using IHHC of the null model and generates 4 models PCV, AIC, and log-likelihood ratio used model selection for a report, which is carried out Stata 14.

Result: The highest risk of both anemia and severe anemia regions are Somalia, Afar, DireDawa, and Harari. Specifically, all zones of Somalia, Afar (zone1, and 3), DireDawa, Harari, and Oromia higher risk of anemia and some of them are high risk for severe anemia. The high concentration of the disease showed in DireDawa, Harari, Jigjiga some part of Gambela and Benishangul Afar at the boundary of Amhara and Tigray and Eastern part of Tigray for anemia. Harari, DireDawa, and Afar at the border of Djibouti had high concentrated(density) severe anemia. Children (Younger age, lower preceding birth interval, stunted, underweight); mothers (younger age and anemic); households (poor and denser family number); and children from the highest and the lowest ecological zone are high risk for anemia. Additionally, children from the younger, work less, uneducated, poor, and anemic mother were high risk of a severe anemia

Conclusion: Across the country, anemia is high, especially rift value areas highly affected by both anemia and severe anemia like Somalia regions and its neighbors. Those regions are not productive areas, lack of health facility, malaria region, backward for any access. So the concerned body must be tackled to minimize this childhood series problem. Before starting any intervention first must be prioritized according to risk, concentration, and characteristics.

\section{Background}

Anemia, defined as a low blood hemoglobin concentration, has been shown to be a public health problem that affects low, middle, and high-income countries [1]. Anemia has a strong influence on children's mental and physical development, including learning, memory, and mental processing speed this consequently affects the latter age of school performance, working capacity [2, 3]; a major cause of child and maternal mortality [4]; increases the chances of fetal deaths, abnormalities, preterm and underweight babies [5]. Short-term consequences of anemia, increased morbidity and mortality, developmental delay, and economic burden for sick children, while long-term consequences are stunted brains and stunted lives, minimize work productivity later in life, and hindering the development of entire societies [6-8]. Therefore, anemia affects not only the individual quality of life, but also the social and economic development of the country, particularly developing countries [79]. According to the World Health Organization (WHO), for under-five children, the threshold $\mathrm{Hb}$ level of being anemic is less than $110 \mathrm{~g} / \mathrm{l}$ [10]. Anemia is considered as severe, moderate, and mild public health problem if its prevalence is $\geq 40 \%$ and $20-39.9 \%, 5-19.9 \%$ respectively else is not anemic in the community $[1,10]$.

In 2011 WHO estimated that about 273.2 million children aged from 6 to 59 months in the world were suffering from anemia (42.6\% of the total anemia), of which 9.6 million are severed anemic and above half $(53.8 \%)$ anemic 
children found in South-East Asia and Africa Regions [1]. Anemia has a higher prevalence in sub-Saharan African (SSA) countries [11]. According to the Ethiopian Demographic and Health Survey (EDHS) report, in Ethiopia, the prevalence of anemia among children 6-59 months old was 53\% in 2005, 44\% in 2011, and 57\% in 2016. From the sampled children in EDHS-2011, 28.6\%, 21.7\%, and 49.7\% were above moderate (both moderate and severe), mildly anemic, and non-anemic, respectively [12]. In all years Ethiopia has severe public health problems in anemia [1]. Even though there was a study about under-5 child anemia in prevalence but not in severe, level, and spatial. There is no study showing the effect of general anemia and high risk (severe) anemia in zonal level and characteristics of child, mother, and condition, and the level of associated factors in Ethiopia among under-5 children based on EDHS-2016. In this study, we investigate the burden of anemia and severe anemia of and associated factors, by level among under-5 children in Ethiopia.

\section{Methods}

\section{Study area}

The study was conducted in Ethiopia, which is located in the North-Eastern part of Africa or known as the "Horn of Africa." It is bounded by the north and south Sudan on the West, Eritrea, and Djibouti on the North East, Somalia on the East and South East, and Kenya to the South. Ethiopia lies between the $3^{0} \mathrm{~N}$ and $15^{0} \mathrm{~N}$ Latitude and $33^{\circ} \mathrm{E}$ and $48^{\circ}$ E Longitude.

The country occupies an area of approximately about $1,127,000 \mathrm{~km}^{2}$, which is almost twice the size of France. The Ethiopian landmass consists of a large, high elevated plateau bisected by the Rift Valley into the northwestern and the southeastern highlands, each with associated lowlands. The contrast in relief is remarkable as land elevation ranges between $-155 \mathrm{~m}$ of Asal Lake in the Afar depression (the lowest point in Africa) to the peak of Mt. Ras-Dejen, at 4,620 meters above sea level in the Semen Mountains [13]. There are nine regional states and two city administrations.

\section{Study design}

A population-based cross-sectional study was employed to explore geographical variation and identify the influencing factors of under-5 child's anemia and severe anemia in Ethiopia.

\section{Data Source}

The data for this study were taken from the 2016 EDHS. The 2016 EDHS is the fourth comprehensive and nationally representative survey conducted in Ethiopia as part of the worldwide Demographic and Health Surveys (DHS) project. The EDHS 2016 data were downloaded from The DHS website after being granted permission.

\section{Dependent Variables: Anemia And Severe Anemia}

The primary outcome of interest was anemia and severe anemia. Anemia is defined as a hemoglobin level of less than the 5th percentile for age and has several causes that vary by age [14]. According to WHO, children under 5 years of age with a hemoglobin level of less than $11.0 \mathrm{~g} / \mathrm{dl}$ is considered anemic. The cutoff values for the various levels of severity were: $<7 \mathrm{~g} / \mathrm{dl}$ for severe anemia, $7 \mathrm{~g} / \mathrm{dl}-9.9 \mathrm{~g} / \mathrm{dl}$ for moderate anemia and $10 \cdot 0 \mathrm{~g} / \mathrm{dl}-10$. $9 \mathrm{~g} / \mathrm{dl}$ for mild anemia [15]. Based on this DHS itself puts individuals final outcome by severity. 


\section{Sample size and sampling procedures}

A weighted sample of 8,482 individuals from 6,460 households, out of which 4084 females and 4398 males were included in the study. All sampling procedures, data collection, and data quality control were done by the DHS team.

\section{Data processing and analysis}

\section{Spatial Statistics Analysis}

Statistically significant clusters defined as geographic areas where the prevalence of the disease is disproportionately higher/lower compared to neighboring areas. Tests for global clustering detect the existence of at least one cluster, but not the specific place of the cluster(s)

\section{Mapping Cluster}

The LISA cluster map shows $p<0.001$ with Moran's I was 0.463 for anemia and 0.306 for severe anemia. The Moran's I indicated clustering patterns (Fig. 3.).

In both panels the red color indicates a high rate of anemia surrounded by high rates of similar cases. Such specific areas were All Somalia region zones except Jigjiga and Shinile, In the Oromia region (Bale, East, and Weast Harergie) was anemia (Fig. 3a); additionally, in Somalia regions, all zones except (Jigjiga, Shinile, and Degahabur) and in Oromia region (Bale and East Harergie) was severe anemia (Fig. 3b).

\section{Hot Spot Analysis}

Hot spot analysis is done through a statistical test. To increase the statistical significance of the result, we used 999 permutations. The red color or high anemia prevalence rates (hot spot) find in Somalia region all zones except Jigjiga; in Oromia region Bale, East and West Harergie; in (South Natinon Nationality People) SNNP region Sidama zone and in Afar region Zone 2 on the border of Eritrea; and Gambela region Zone 3 at the edge of the border (Figur 4a). The hot spots of high severe anemia prevalence rates locates in all zones of the Somalia region except Jigjiga; in Oromia region Bale and East Harergie zones (Figur 4b).

\section{Heat Map}

In Fig. 5. The heat map shows the concentration of anemia and severe anemia in a specific area. For anemia, the concentrated area or location ware Harari and DireDawa and their surrounding, north direction of Gambela region at the border, North-East of SNNP region at the boundary of Oromia region, South direction of Benishangul Gumz region at the border with the boundary of Oromia region and most part of Tigray region high anemia concentration in their specific area or a higher density of anemia in this area (Fig. 5a). According to severe anemia Dire Dawa and Hararie with their surrounding, Somalia region in the boundary of the Oromia region in the direction 
of Hareri and dire Dawa and Afar region at the border of Djibouti, where higher density of severe anemia compared to other areas.

\section{Kulldoruff's Scan Statistic}

Kulldoruff's scan statistic is a spatial scan statistics method for detecting and evaluating statistically significant spatial clusters risk factors for a specific disease. Final confirmatory spatial analyses were done using spatial analysis software SaTScan and QGIS. The SaTScan declares where spatially significant higher an lower rates of aggregates are found. Its output presents the hotspot areas in circular windows, indicating the areas in the windows are higher than expected distributions compared to the areas outside of the cluster windows [19-22].

\section{Multilevel Logistic Regression}

Multilevel logistic regression was utilized to assess the impact of measured lower-level and higher-level factors. Multilevel analysis was considered appropriate in order to account for the hierarchical nature of the EDHS data and to be able to estimate household-level effects on the outcome variable [23-25].

\section{Model Building}

The four model building and analysis were done using STATA14. Overall, four models containing variables of interest were fitted for each of the under- 5 anemic and severe anemic children. The first model $\left(M_{0}\right)$ is an empty model which was fitted without independent variables to test random variability using Intra House Hold Correlation (IHHC) [26]. The second model $\left(\mathrm{M}_{1}\right)$ was fitted to all lower-level (individual level) factors; the third model $\left(M_{2}\right)$ used for all higher-level (household level) factors; and the fourth model $\left(M_{3}\right)$ used for both lower- and higher-level factors to report. Model fitness for the report selected by the Akaike Information Criterion (AIC).

\section{Result}

\section{Spatial Epidemiology Of Anemia By Severity}

The prevalence of anemia by severity across the country was severe (3.1\%), moderate (29.5), and mild (25.1\%). The five highest regions with childhood anemia were Somalia (83.5\%), Afar (74.7\%), DireDawa (72.1\%), Harari (66.8\%), and Oromia (66.1\%). The five highest severe childhood anemia regions were Somalia (12.4\%), DireDawa (9.6\%), Harari (5.4\%), Afar (4.2\%), and Oromia (3.8\%) (Fig. 1).

\section{Risk Of Anemia And Severe Anemia Per 1000 Children}

The left panels (anemia) of the red color indicates above 800 anemia cases per 1000 children, Those zones are in Somalia region Worder (872), Jigjiga (864), both Korahe and Gode (846 each), and Afder (808) zones; In the Afar region Zone 1 (810) and Zone 2 (809) at the boundary of Tigray; In Oromia region East Harergie (801) zone; and in the Gambela region on the left of ZONE 1 (873). The yellow color indicates the zones, risk of anemia between 600 and 800 , this second burden lies all zones in Oromia region in the direction of the South-East (from Addis Abeba 
to Border of Somalia); Northern and Southern edge of Afar region; In Gambela region all zones those have common boundaries with Oromia region; and Degehabur In Somalia region (Fig. 2a). The right panel identifies the risk of severe anemia per 1,000 children in each zone of the country. The red color indicates above 90 cases of severe anemia per 1000 children, those zones are in Somalia region Worder (106), Jigjiga (161), Korahe (115), and Gode (103); and Oromia region only Borena zone (104). The yellow color indicates zones severe anemia burden lays 60 to 90 per 1000 children. White colors indicate lake and unmeasured areas by EDHS (Fig. 2b).

\section{Mapping Cluster}

The LISA cluster map shows $p<0.001$ with Moran's I was 0.463 for anemia and 0.306 for severe anemia. The Moran's I indicated clustering patterns (Figure 3.).

In both panels the red color indicates a high rate of anemia surrounded by high rates of similar cases. Such specific areas were All Somalia region zones except Jigjiga and Shinile, In the Oromia region (Bale, East, and Weast Harergie) was anemia (Figure 3a); additionally, in Somalia regions, all zones except (Jigjiga, Shinile, and Degahabur) and in Oromia region (Bale and East Harergie) was severe anemia (Figure 3b).

\section{Hot Spot Analysis}

Hot spot analysis is done through a statistical test. To increase the statistical significance of the result, we used 999 permutations. The red color or high anemia prevalence rates (hot spot) find in Somalia region all zones except Jigjiga; in Oromia region Bale, East and West Harergie; in (South Natinon Nationality People) SNNP region Sidama zone and in Afar region Zone 2 on the border of Eritrea; and Gambela region Zone 3 at the edge of the border (Figur 4a). The hot spots of high severe anemia prevalence rates locates in all zones of the Somalia region except Jigjiga; in Oromia region Bale and East Harergie zones (Figur 4b).

\section{Heat Map}

In figure 5. The heat map shows the concentration of anemia and severe anemia in a specific area. For anemia, the concentrated area or location ware Harari and DireDawa and their surrounding, north direction of Gambela region at the border, North-East of SNNP region at the boundary of Oromia region, South direction of Benishangul Gumz region at the border with the boundary of Oromia region and most part of Tigray region high anemia concentration in their specific area or a higher density of anemia in this area (Figure 5a). According to severe anemia Dire Dawa and Hararie with their surrounding, Somalia region in the boundary of the Oromia region in the direction of Hareri and dire Dawa and Afar region at the border of Djibouti, where higher density of severe anemia compared to other areas.

\section{Satscan}

A total of 5 for anemia and 8 for severe anemia SaTScan clusters were identified in the order of child anemia and severe anemia orderly from the SaTScan spatial analysis, but only 2 for anemia and 5 for severe anemia of them were statistically significant circular windows. The SaTScan spatial analysis result detected the most likely 
primary spatial clusters for anemia all zones Somalia regions except Shinile and for severe anemia Jigjiga zone in Somalia region. The most likely secondary clusters for child anemia was observed from Zone 1 \& 3 in afar region and for child severe anemia Liben in Somalia and Borena in Oromia region. The remaining clusters of severe anemia regions at the 3rd, 4th, and 5th stage were East Harergie in Oromia, Gode, Korahe Worder, Afder in Somalia, and Zone 1 in Afar region (Fig. 6, Table 1).

Table 1

SaTScan spatial analysis of anemia and severe anemia in Ethiopia

\begin{tabular}{|c|c|c|c|c|c|}
\hline Cluster & Radius & Zone & LLR & RR & P-value \\
\hline \multicolumn{6}{|l|}{ Anemia } \\
\hline 1 & 511 & Somalia except Shinile & 45.6 & 1.4 & $<0.001^{\star \star}$ \\
\hline 2 & 145 & Zone $1 \& 3$ (Afar) & 13.2 & 1.4 & $<0.001^{\star \star}$ \\
\hline 3 & 29 & Zone 1(Gambela) & 5.6 & 1.5 & 0.633 \\
\hline 4 & 111 & Arsi and East Shoa & 3.7 & 1.2 & 0.994 \\
\hline 5 & 97 & Borena & 3.5 & 1.6 & 0.998 \\
\hline \multicolumn{6}{|c|}{ Severe anemia } \\
\hline 1 & 59.6 & Jigjiga & 23.9 & 4.9 & $<0.001^{\star \star}$ \\
\hline 2 & 247 & Borena and Liben & 23.1 & 6 & $<0.001^{\star *}$ \\
\hline 3 & 26 & East Harergie, Gode, Korahe & 20.1 & 5.6 & $<0.001^{\star *}$ \\
\hline 5 & 41.6 & Zone 1(Afar) & 11.7 & 5.3 & $0.001^{\star \star}$ \\
\hline 6 & 12.4 & DireDawa & 6.4 & 3.5 & 0.224 \\
\hline 7 & 7.34 & Harari & 5.1 & 3.4 & 0.619 \\
\hline \multirow[t]{2}{*}{8} & 3.9 & Harari & 4.4 & 3.3 & 0.859 \\
\hline & ** stati & cally significant at $p<0.05$ & & & \\
\hline
\end{tabular}

\section{Prevalence And Multilevel Logistic Regression}

From EDHS 2016 Women/Individual data set, a total of 8482 children aged 6-59 months where the primary outcome (hemoglobin level) was included in the analysis, of which 4,084 (48\%) were female and 4,398 (52\%) were male. Out of the total 8,482 samples $57.6 \%(4,882)$ individuals were anemic; of which $3.1 \%(262)$ were severe anemic child [See Additional file 1].

\section{Model Comparison}

In this study $\mathrm{IHHC}$ value was $16.95 \%$ and $13.35 \%$ for anemic and severely anemic respectively, which is $>5 \%$, which indicates the level analysis is more appropriate than a binary logistic regression model. Both The AIC and $\mathrm{BIC}$ calculate model fitness for the report, which indicates the smallest $\mathrm{M}_{3}$ (both individual and household level) 
for anemic and $\mathrm{M}_{2}$ (only household level) for sever anemic was the best model fitness for the final report (Table 2).

Table 2

Model comparison for report among $\mathrm{M}_{0}, \mathrm{M}_{1}, \mathrm{M}_{2}$ and $\mathrm{M}_{3}$

\begin{tabular}{|c|c|c|c|c|c|c|c|c|}
\hline \multirow{2}{*}{$\begin{array}{l}\text { Random } \\
\text { effect }\end{array}$} & \multicolumn{2}{|c|}{ Empty $\left(M_{0}\right)$} & \multicolumn{2}{|c|}{ Individual $\left(\mathrm{M}_{1}\right)$} & \multicolumn{2}{|c|}{ Household $\left(\mathrm{M}_{2}\right)$} & \multicolumn{2}{|l|}{ All $\left(M_{3}\right)$} \\
\hline & \multirow[t]{2}{*}{ Anemia } & Severe & \multirow[t]{2}{*}{ Anemia } & Severe & \multirow[t]{2}{*}{ Anemia } & Severe & \multirow[t]{2}{*}{ Anemia } & \multirow{2}{*}{$\begin{array}{l}\text { Severe } \\
\text { Anemia }\end{array}$} \\
\hline & & Anemia & & Anemia & & Anemia & & \\
\hline Variance & 19.92 & 3.32 & 17.1 & 3.16 & 19.74 & 3.27 & 16.81 & 3.13 \\
\hline $\mathrm{IHHC}(\%)$ & $16.95^{\star}$ & $13.35^{\star}$ & 20.83 & 13.24 & 12.13 & 10.8 & 18.83 & 11.01 \\
\hline PCV & Refer. & Refer. & 14.16 & 4.82 & 0.9 & 1.51 & 15.6 & 4.22 \\
\hline $\begin{array}{l}\text { Log } \\
\text { likelihood }\end{array}$ & -5469 & 1671 & -3951 & 1397 & $-4,871$ & $1747^{\star \star}$ & -3624 ** & 1,431 \\
\hline AIC & 10945 & -3336 & 7926 & -2770 & 9791 & $-3459 * \star$ & $7315^{\star \star}$ & -2809 \\
\hline $\mathrm{BIC}$ & -3336 & -3315 & -2770 & -2690 & -3459 & $-3334^{* *}$ & $7534^{\star *}$ & -2628 \\
\hline
\end{tabular}

\section{Multilevel Logistic Regression For Under-5 Child's Anemia}

After controlling another individual and household level factors; When the child age increase by a month the risk of stunting decreased by $10 \%(A O R=0.9, \mathrm{Cl}$ : $0.89-0.92)$. Stunted children 2.18 times higher risk of anemia (AOR $=2.18, \mathrm{Cl}: 1.58-3.00)$ as compared to whose counterparts. Wasted children, $63 \%$ more risk of anemia $(A O R=1.63$, Cl: 101-2.65). Ever-breastfed but not currently breastfeeding children were $52 \%(\mathrm{AOR}=0.48, \mathrm{Cl}: 0.33-0.69)$ more likely to be anemic than still breastfeeding. Underweight children were $60 \%$ more likely to risk for anemia than normal and above. When a family household size increased by 1 person the risk of anemia in child increased by $19 \%(A O R=1.19, \mathrm{Cl}: 1.08-1.31)$. The mother's age increased by one year the risk of anemia on the child decreased by $6 \%(A O R=0.94, \mathrm{Cl}: 0.91-0.97)$. From poor family child $76 \%$ more risk than from rich family child $(A O R=1.76, \mathrm{Cl}: 1.15-2.69)$. Female household head family child 2.14 times exposed by anemia compared to their counterparts within $(A O R=2.14, \mathrm{Cl}: 1.27-3.61)$. Children whose mother anemia status mild, moderate, and severe were 4.18, 4.29, and 7.88 times more likely to be anemic ( $\mathrm{AOR}=4.18, \mathrm{Cl}$ : $2.78-6.3)$; $(\mathrm{AOR}=4.29, \mathrm{Cl}$ : 2.21-8.35); and $(\mathrm{AOR}=7.88, \mathrm{Cl}: 1.75-35.61)$. Household who lives in subtropical was $8.45(\mathrm{AOR}=8.45, \mathrm{Cl}$ : 2.91-24.51) and in highland $3.14(\mathrm{AOR}=3.14, \mathrm{Cl}$ : 2.1-4.7) were times higher risk of their child with anemia than whose household who lives in temperate regions [See Additional file 1].

\section{Multilevel Logistic Regression For Under-5 Children Severe Anemia}

After controlling other household-level factors; Mother age, mother education status, household wealth, mother anemia status, mother working status, were statically significant factors for severe anemia. Mother's age 
increased by one year the risk of severe anemia on the child decreased by $3 \%(A O R=0.97, \mathrm{Cl}: 0.915-0.99)$. Children born from uneducated women, $38 \%$, (AOR $=0.62, \mathrm{Cl}: 0.42-0.92)$ more risk for severe anemia than a child born from primary educated mother. Children whose mother anemia status mild, moderate, and severe were 2.46, 4.5 and 9.38 times more likely to be severe anemic ( $\mathrm{AOR}=2.46, \mathrm{Cl}: 1.76-3.45)$; $\mathrm{AOR}=4.5, \mathrm{Cl}$ : $3.0-6.75)$; and $(A O R=9.38, \mathrm{Cl}: 4.67-18.87)$ respectively. Women who have not worked their child $42 \%$ higher risk of severe anemia within $(A O R=0.58, \mathrm{Cl}: 0.40-0.84)$ compared to children whose mother has worked. Children get from poor household $2.28(\mathrm{AOR}=2.28, \mathrm{Cl}$ : 1.41-3.67) times higher risk of severe anemic compared to children of the rich household [See Additional file 1].

\section{Discussion}

Different environmental conditions play different roles in the anemia and severe anemia burden. In different geographical regions have different burdens of anemia.

Based on the WHO standard (anemia prevalence $>40 \%$ is a severe public health problem) [1], so Ethiopia is categorized as severe public health problem regions because its prevalence is $57.6 \%$. Epidemiology of anemia across administrative regions indicates in non-stationary nature (i.e. Spatial variation in spatial dependence), this variation may occur because of human-induced environmental transformations, the spatial variation, topography, and burdens of different disease and its implementation of disease control. Our results show that geographical variation is the main anemia causing factors. Overall anemic the highest distribution from the top are Somalia, Afar, DireDawa, Harari, and Oromia regions are highly affected regions their distribution is laid between the range of $66.1-83.5 \%$ those are above country average distribution of childhood anemia. The highest affected areas or children with severe anemia regions orderly with the highest are Somalia, DireDawa, Harari, Afar and Oromia their distribution is in the range of 3.8-12.4\%. In the general overall distribution of childhood, anemia is high almost half of the regions were indicating higher than country distribution level, which is Somalia, Afar, DireDawa, Harari, and Oromia.

All spatial analysis results show a high risk in a similar place. Which indicates mainly affect areas in anemia or hot spot areas in anemia are all zones of Somalia and most zones of Oromia in the direction of additionally, Blae, Borena, and East Harerge were a hot spot for severe anemia; All zones of Afar region are showing lie on the burden but Zone 1 indicated severe anemia; In Gambela region both Zone 1 and zone 3 have hotspot zones for anemia; Dire Dawa and Harari have the burden of anemia and severe anemia. The above region of the country contributes the highest-burden of anemia the list of possible reason are: This region is not productive and have not owned source of plant product to find enough complementary feeding; Most of this region is the subtropical region in this region anemia prevalence is high [27]; These regions are malaria-affected region, malaria was the leading factor associated with anemia [28]. Most of this region the primary, complementary food is animal milk, which is poor sources of iron or negative effect on non-heme and heme iron absorption [29-31]. The other tangible reason peoples who live in lowland widely dispersed and they can't get health facility and road (transport), so they can't find any intervention like iron and folic acid and have accesses advice of neither before nor after delivery. Those peoples' lives are bound up with animal-like goat, cow, and camel so they are pastoral, the first problem is this type of work the person who has no permanent residence throughout their life depend on other factors like the accesses of water and forage for their animals, so must move from place to place and the second problem this pastoral job is culturally done by females, both during pregnancy and after birth, sometimes the women will be born on work, so the mother and her child ride different burdens like a hard bit of sunlight, 
hanger and they are not finding rest and care. The other region is a relatively low risk of anemia; this is due to the potential of producing teff, which is iron-rich food. The lowest distribution of anemia regions is like Amhara and Benishangul Gumz but the surprising thing is these lowest regions are according to WHO lie on sever public health problem regions. So, relatively can be said high and low hot spot and cold spot but in fact all the regions of the country under severe public health problems [1].

Based on the geographical variation result, Anemia and severe anemia with respect to the specific area like zone indicates. The densest distribution of anemia with a narrow area is located in Harari and Dire Dawa and surrounded, some part of Gambela, S-W of Benishangul Gumz, North-East direction of the country especially boundary of Tigray with Afara and Amhara with Afar. And for severe anemia, high denser shows Afar region at the border of Djibouti, DireDawa and Harari and their surrounding and Jigjiga zone in Somalia region in comfort areas for treatment and other intervention for many children with a limited resource and man-power because the child on the anemia burden finds clothes each other, but the other hot spot areas the peoples are live scattered as well as the case is scattered like Somalia region so it is not easily manageable, which means they need more resource and man-power for tackling the problem.

Maternal (anemia status and age) and household wealth are show significant contribution for both anemia and severe anemia prevalence; Mother working and educational status are a significant contribution for only severe anemia, But any factor does not contradict between anemia and severe anemia. Maternal anemia directly related to both child anemia and severe anemia. This finding strongly agrees with another finding like a study done in Ethiopia DHS-2011 [32], in Togo DHS 2013-14 [33], in Malawi DHS-2010 [34]. This strong association could be due to many factors that determine anemia because many things share between mother and child such as worm infection, malaria, and other infectious diseases that affect red blood cell production and iron stores [35]. Likewise, low levels of iron, zinc, folate, and vitamin A and B12 in the breast milk of the anemic mother could also affect the Hemoglobin level of the breastfeeding child. Younger children high risk for anemia and severe anemia whose mother anemic during pregnancy [36]. The other reason may be the child finds enough iron stores in their bodies at least the first six months from his/her mother during lactation.

The other factor strong association with both anemia and severe anemia is the wealth status of the family. The child with poor family highly affected by anemia and severe anemia this output in line with other findings done in different areas [37-40]. This finding could be due to the reason for problems in production or purchasing necessary food for themselves and their children, so they can not get iron-rich foods. The other reason poor mothers might have problems in purchasing and providing good nutritious which might result in anemia. This will be a fact because, child below 12 months and their mothers share a similar sociological environment, their dietary patterns, and their quality of life similar.

Children high risk for anemia and severe anemia who are from younger mothers for anemia and severe anemia. this results in the same as others like research done Ethiopia[38], in Bangladesh [41] and in Sub Sahara Africa [42]. The reason for child feeding, hygiene, and child care older age mothers have good experienced. And the other result older women are economically developed and the child has easily gained iron-rich foods.

Child age increases the risk of anemia decrease this result similar with other findings such as a study done in Ethiopia Eastern Amhara [38], EDHS-2011 [12], and in Sub Sahara Africa [42] this could be explained by the fact that older children receive a diet that is richer and complete iron, with sufficient intake of iron it prevents the occurrence of anemia in the child.

Page $10 / 18$ 


\section{Conclusion}

This finding identifies based on different dimensions which are based on sample populations (prevalence, hotspot, association with neighbors), based on specific area density (accumulation), based on child characteristics, and maternal characteristics with some community characteristics. So anybody easily understands the situation with other characteristics supporting or reducing this situation and can be intervention easily based on this finding. Specialty planners and decision makers easily understand the disease situation and to identify the type of intervention based on place, characteristics, severity, accumulation. And additionally, researchers can be further analysis this identifies the place and group why/how are high risks and how to minimize this problem. Generally, anemia is high, especially rift value areas highly affected by both anemia and severe anemia especially Somalia regions and its neighbors. Those regions are not productive areas, lack of health facility, malaria region, backward for any access. So the concerned body must be tackled to minimize this childhood series problem. Before starting any intervention first must be prioritized according to risk, concentration, and characteristics.

\section{Abbreviations}

AOR: Adjusted Odds Ratio; Cl:Confidence Interval; DHS:Demographic and Health Survey; EDHS:Ethiopia Demographic and Health Survey; IHHC:Intra House Hold Correlation; SD:Standard Deviation; SNNP:South Nations Nationality People; WHO:World Health Organization; AIC; Akaike Information Criterion; BIC:Bayesian Information Criterion; PCV:Proportional Change in Variance; CSA:Central Statistical Agency ; LISA:Local Indicators of Spatial Association

\section{Declarations}

\section{Ethics approval and consent to participate}

Ethical clearance with written consent (AuthLetter_130669) was obtained from Measure DHS International Program which authorized the data sets. All the data which used in this study are publicly available, aggregated secondary data with not having any personal identifying information that can be linked to particular individuals, communities, or study participants. Confidentiality of data maintained anonymously.

\section{Consent for publication}

Not Applicable

\section{Availability of data and materials}

The data set we used which is the '2016 Ethiopian Demographic and Health Survey' were obtained from the DHS program (www.dhsprogram.com), but the 'Dataset Terms of Use' do not permit us to distribute this data as per data access instructions (http://dhsprogram.com/data/Access-Instructions.cfm). To get access for the dataset you must first be a registered user of the website (www.dhsprogram.com), and download the 2016 Ethiopian Demographic and Health Survey datasets. 


\section{Competing interests}

The authors declare that they have no conflicts of interest.

\section{Funding}

There was no any funding or sponsoring organization for this paper.

\section{Authors' contributions}

BAH conceived the general research design and participated in data analysis, interpretation and wrote the first and final draft. GGB coordinated the study, reviewed the manuscript and contributed with critical comments and drafted the manuscript. BAM coordinate the spatial part of the study and comments and drafted the manuscript based on geographical characteristics and comment the whole document. BMG contributes the comment on the statistical part of the paper and comment final manuscript. JB participated in research design and refined the general research idea, reviewed the manuscript, and contributed comments.

All authors read, approved and contributed with critical comments and drafted the manuscript final paper.

\section{Acknowledgements}

The authors are sincerely grateful to the Central Statistical Agency (CSA) and The Demographic Health System (DHS) program for providing us to use the 2016 EDHS dataset through their archives (archive@dhsprogram.com)

\section{References}

1. Organization WH, Control CfD, Prevention. The global prevalence of anaemia in 2011. Geneva: WHO; 2015.

2. Soliman AT, De Sanctis V, Kalra S. Anemia and growth. Indian journal of endocrinology metabolism. 2014;18(Suppl 1):S1.

3. Grantham-Mcgregor S, Ani C. Iron-deficiency anemia: reexamining the nature and magnitude of the public health problem. J Nutr. 2001;131(2):649S-668S.

4. Marn H, Critchley JA. Accuracy of the WHO Haemoglobin Colour Scale for the diagnosis of anaemia in primary health care settings in low-income countries: a systematic review and meta-analysis. The Lancet Global Health. 2016;4(4):e251-65.

5. Saikia N, et al. Trends in geographical mortality differentials in India. Rostock: Max Planck Institute for Demographic Research; 2009.

6. De Onis M, Branca F. Childhood stunting: a global perspective. Matern Child Nutr. 2016;12:12-26.

7. Anaemias WN, Tools for Effective Prevention and Control. World Health Organization, 2017.

8. Balarajan Y, et al. Anaemia in low-income and middle-income countries. The lancet. 2011;378(9809):212335.

9. Zavaleta N, Astete-Robilliard L. Effect of anemia on child development: Long-term consequences. Revista peruana de medicina experimental y salud publica, 2017. 34(4): p. 716-722. 
10. Organization $\mathrm{WH}$, Haemoglobin concentrations for the diagnosis of anaemia and assessment of severity, 2011, World Health Organization.

11. Stevens GA, et al. Global, regional, and national trends in haemoglobin concentration and prevalence of total and severe anaemia in children and pregnant and non-pregnant women for 1995-2011: a systematic analysis of population-representative data. The Lancet Global Health. 2013;1(1):e16-25.

12. Demographic CE. Health Survey-2011. Central Statistical Agency Addis Ababa. Ethiopia ICF International Calverton, Maryland, USA. 2012, 2016.

13. Billi P. Geomorphological landscapes of Ethiopia. Landscapes and landforms of Ethiopia, 2015: p. 3-32.

14. Janus J, Moerschel SK. Evaluation of anemia in children. Am Family Phys. 2010;81(12):1462-71.

15. Benoist B, et al., Worldwide prevalence of anaemia 1993-2005: WHO global database on anaemia. Worldwide prevalence of anaemia 1993-2005: WHO global database on anaemia, 2008.

16. Anselin L, Exploring spatial data with GeoDaTM: a workbook. Center for spatially integrated social science, 2005.

17. Anselin L, Syabri I, Kho Y, GeoDa: an introduction to spatial data analysis, in Handbook of applied spatial analysis. 2010, Springer. p. 73-89.

18. Yeap E, Uy I, Marker clustering and heatmaps: New features in the Google Maps Android API utility library. Google Geo Developers, 2014.

19. Kulldorff M, Nagarwalla N. Spatial disease clusters: detection and inference. Statistics in medicine. 1995;14(8):799-810.

20. Jacquez GM, Greiling DA. Local clustering in breast, lung and colorectal cancer in Long Island, New York. International Journal of Health Geographics. 2003;2(1):3.

21. JACQUEZ GM. Statistical software for the clustering of health events. Statistics in medicine. 1996;15(79):951-2.

22. Huang L, Pickle LW, Das B. Evaluating spatial methods for investigating global clustering and cluster detection of cancer cases. Statistics in medicine. 2008;27(25):5111-42.

23. Rabe-Hesketh S, Skrondal A, Multilevel and longitudinal modeling using Stata. 2008: STATA press.

24. Goldstein H, Multilevel statistical models. Vol. 922. 2011: John Wiley \& Sons.

25. Hox JJ, Moerbeek M, Van de Schoot R, Multilevel analysis: Techniques and applications. 2017: Routledge.

26. Diez R. A glossary for multilevel analysis. J Epidemiol Commun Health. 2002;56(8):588.

27. Harding KL, et al. Determinants of anemia among women and children in Nepal and Pakistan: An analysis of recent national survey data. Matern Child Nutr. 2018;14:e12478.

28. Ouédraogo $S$, et al. Maternal anemia at first antenatal visit: prevalence and risk factors in a malaria-endemic area in Benin. Am J Trop Med Hyg. 2012;87(3):418-24.

29. Hallberg $L$, et al. Bioavailability in man of iron in human milk and cow's milk in relation to their calcium contents. Pediatr Res. 1992;31(5):524-7.

30. Levy-Costa RB, Monteiro CA. Cow's milk consumption and childhood anemia in the city of São Paulo, southern Brazil. Revista de saude publica. 2004;38:797-803.

31. Vanderhoof JA, Kleinman RE. Iron requirements for infants with cow milk protein allergy. J Pediatr. 2015;167(4):S36-9. 
32. Muchie KF. Determinants of severity levels of anemia among children aged 6-59 months in Ethiopia: further analysis of the 2011 Ethiopian demographic and health survey. BMC Nutrition. 2016;2(1):51.

33. Nambiema A, Robert A, Yaya I, Prevalence and risk factors of anemia in children aged from 6 to 59 months in Togo: analysis from Togo demographic and health survey data, 2013-2014. BMC public health, 2019. 19(1): p. 215.

34. Ngwira A, Kazembe LN. Bayesian random effects modelling with application to childhood anaemia in Malawi. BMC Public Health. 2015;15(1):161.

35. Brooker S, et al. Epidemiology of plasmodium-helminth co-infection in Africa: populations at risk, potential impact on anemia, and prospects for combining control. Am J Trop Med Hyg. 2007;77(6_Suppl):88-98.

36. Abu-Ouf NM, Jan MM. The impact of maternal iron deficiency and iron deficiency anemia on child's health. Saudi Med J. 2015;36(2):146.

37. Gebreweld A, et al., Prevalence of anemia and its associated factors among children under five years of age attending at Guguftu health center, South Wollo, Northeast Ethiopia. PloS one, 2019. 14(7).

38. Engidaye G, et al. Under nutrition, maternal anemia and household food insecurity are risk factors of anemia among preschool aged children in Menz Gera Midir district, Eastern Amhara, Ethiopia: a community based cross-sectional study. BMC Public Health. 2019;19(1):968.

39. Woldie $\mathrm{H}$, Kebede $\mathrm{Y}$, Tariku A, Factors associated with anemia among children aged 6-23 months attending growth monitoring at Tsitsika Health Center, Wag-Himra Zone, Northeast Ethiopia. Journal of nutrition and metabolism, 2015. 2015.

40. Kawo KN, Asfaw ZG, Yohannes N, Multilevel analysis of determinants of anemia prevalence among children aged 6-59 Months in Ethiopia: classical and bayesian approaches. Anemia, 2018. 2018.

41. Khan JR, Awan N, Misu F. Determinants of anemia among 6-59 months aged children in Bangladesh: evidence from nationally representative data. BMC Pediatr. 2016;16(1):3.

42. Moschovis PP, et al. Individual, maternal and household risk factors for anaemia among young children in sub-Saharan Africa: a cross-sectional study. BMJ open. 2018;8(5):e019654.

\section{Figures}




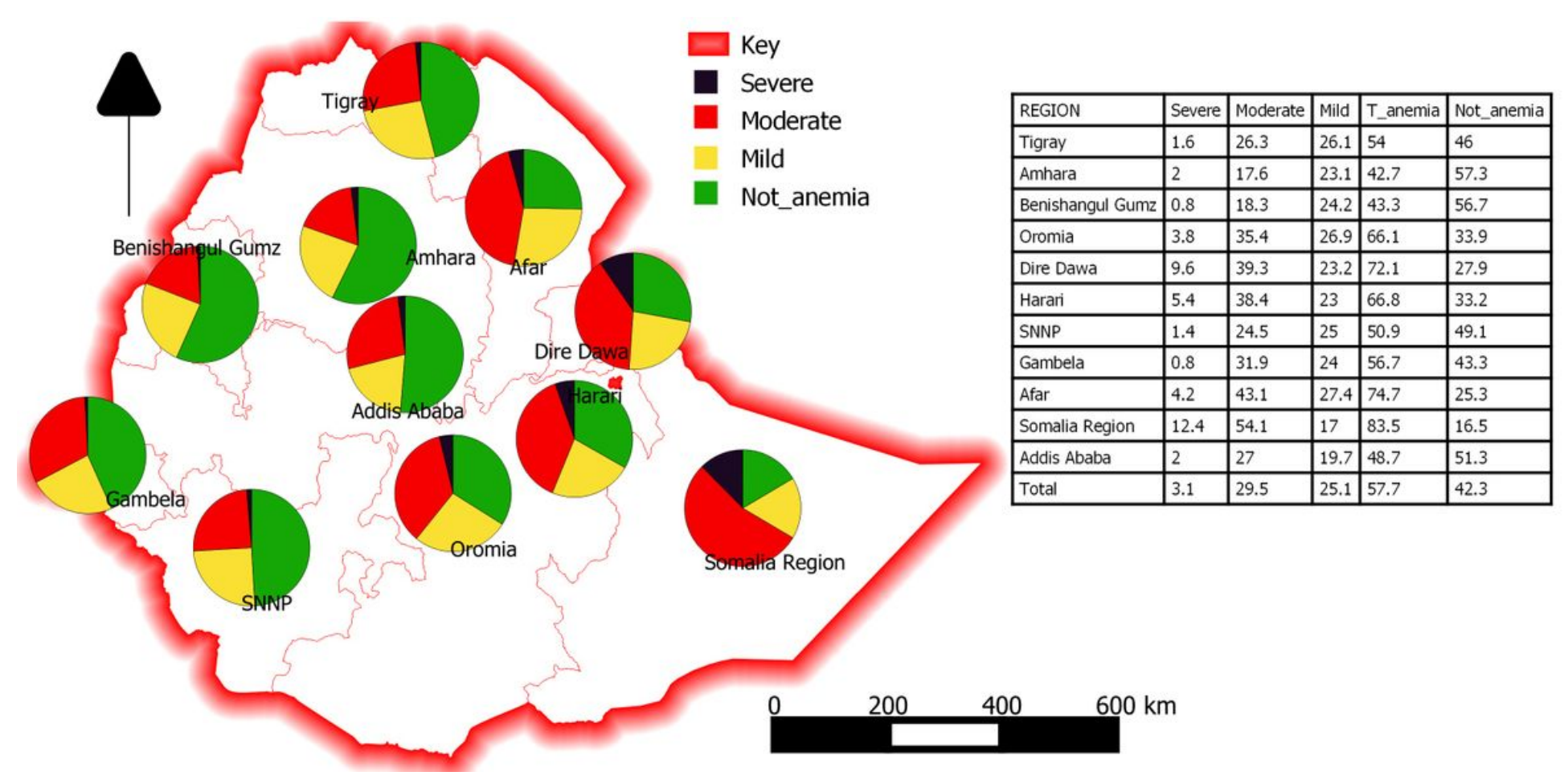

\section{Figure 1}

Prevalence of anemia by severity and region in Ethiopia, 2016. The pie-chart of the black color indicates severe (< $7 \mathrm{~g} / \mathrm{dl})$, red color for moderate $(7 \mathrm{~g} / \mathrm{dl}-9.9 \mathrm{~g} / \mathrm{dl})$, yellow color for mild $(10 \cdot 0 \mathrm{~g} / \mathrm{dl}-10 \cdot 9 \mathrm{~g} / \mathrm{dl})$, and the green color for not anemia( $(\geq 11 \mathrm{~g} / \mathrm{dl})$. To carry out this analysis used QGIS 3.4.3, which available at https://www.npackd.org/p/qgis/3.4.3.

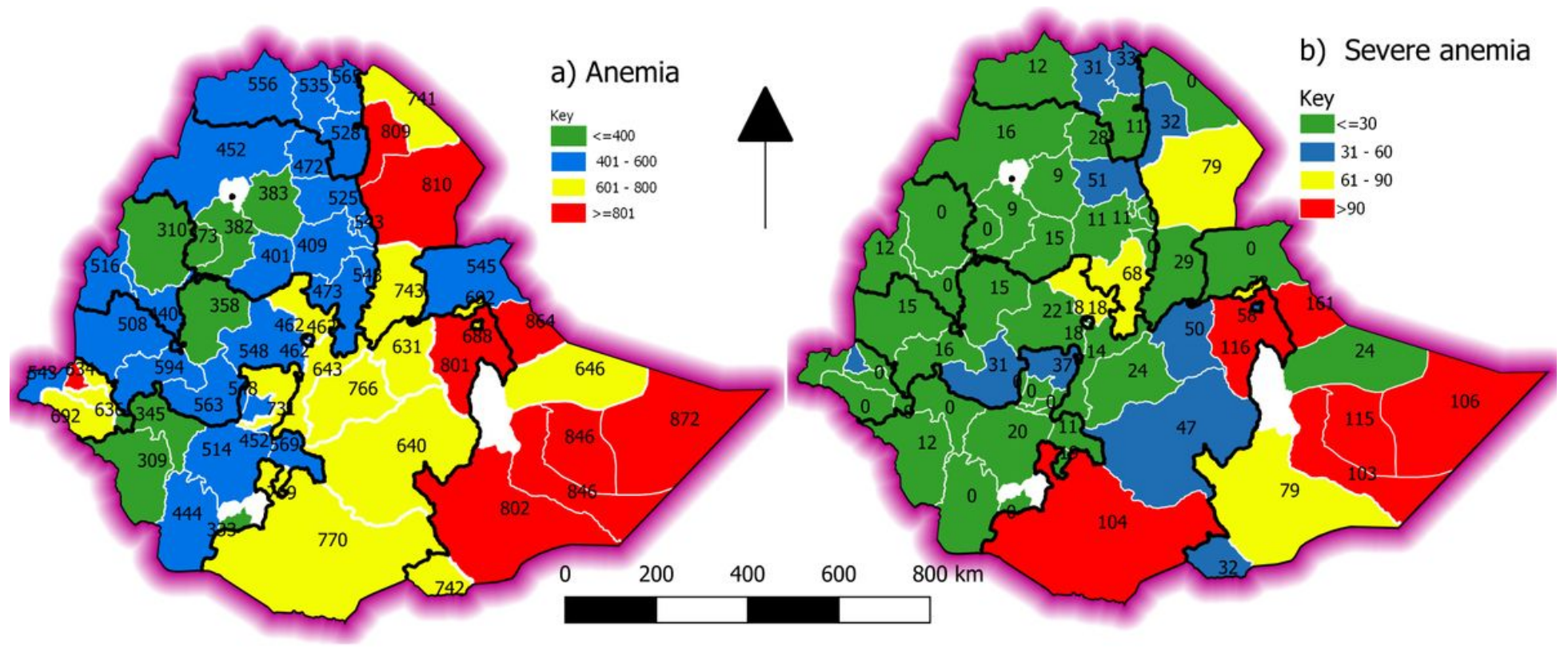

\section{Figure 2}

Risk of anemia and severe anemia per 1000 children in Ethiopia, 2016. The left panel (a) indicates anemia the right (b) is severe anemia. The red color indicates the risk of per 1000 children is anemia above 800 and above 90 severe anemia. The yellow color indicates 401-600 for anemia and 61-90 for severe anemia. The blue color 
indicates 401-600 for anemia and 31-60 for severe anemia The green color indicates below 400 for anemia and below 30 for severe anemia. The white color indicates one of the in the direction of North is Tana lake the rust is the zone from which DHS did not take a sample. To carry out this analysis used QGIS 3.4.3, which available at https://www.npackd.org/p/qgis/3.4.3.

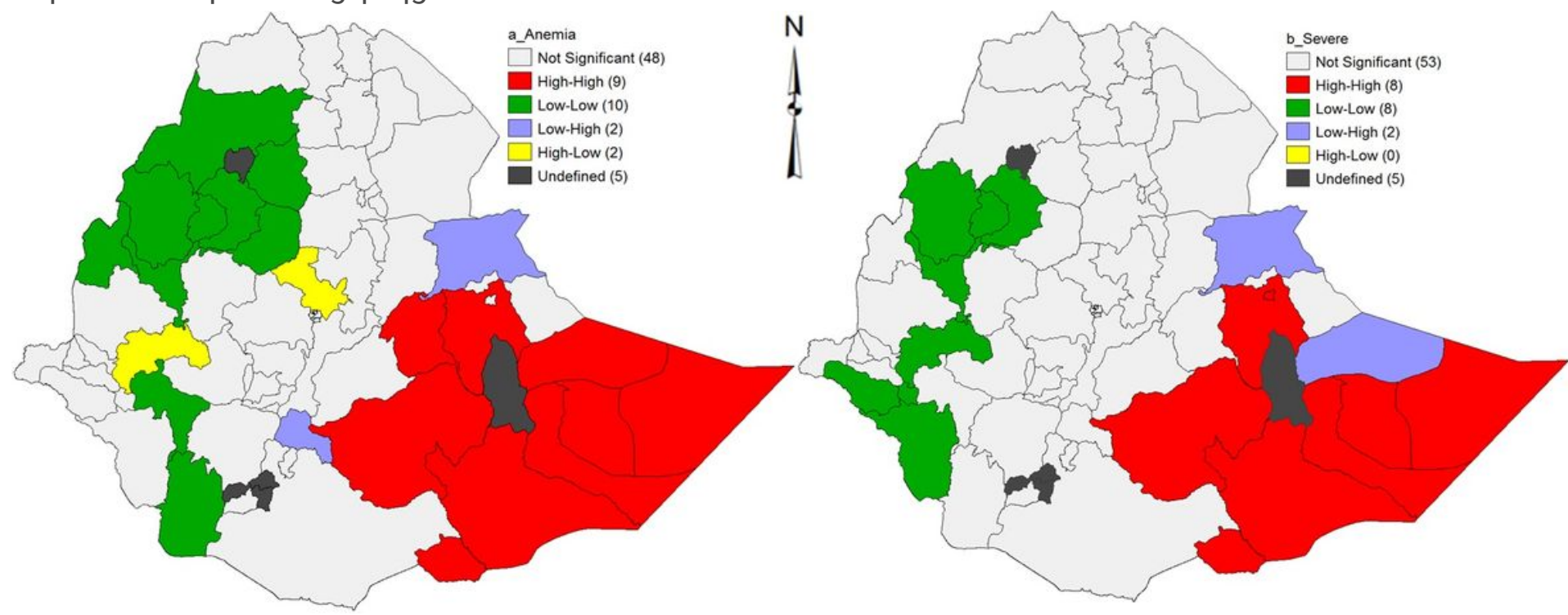

\section{Figure 3}

LISA cluster map of anemia and severe anemia in Ethiopia, 2016 The left side of the map indicates child anemic and the right side of the map indicates severe anemia. Each polygon on the map represents a single zone area with a burden of anemia and severe anemia in children. High-High (red color) means high rates of anemia and severe anemia children surrounded by similar characteristics; low-low (green color) means low rates of anemia and severely anemic children surrounded similar characteristics. Low-High (Dark-Blue color) indicates low rates of anemia and severely anemic children surrounded by high rates of anemia and severe anemia in children. High-low (yellow color) means high rates of anemia and severe anemia children surrounded by low rates of anemia and severe anemia children; undefined (black color) indicates lakes and zones (not taken sample in this area). The red color indicates hotspot areas of anemia and severe anemia; the green color indicates cold-spot areas of anemia and severe anemia; and the yellow and dark-blue colors show outliers. The hotspots are public health importance. To conduct this analysis use GeoDa version 1.14, which is available at: https://geodacenter.github.io/download_windows.html. 


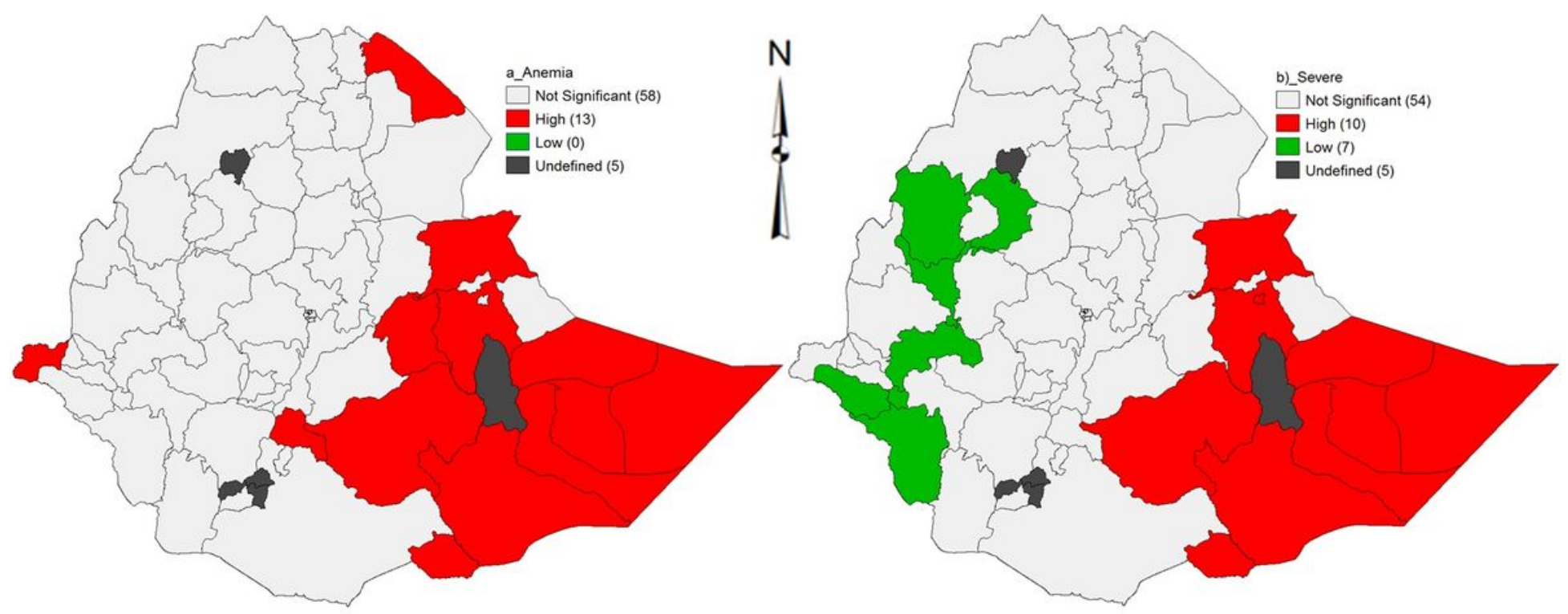

\section{Figure 4}

Hotspot map of anemia and severe anemia in Ethiopia, 2016 The left side of the map indicates anemia and the right side of severe anemia. Each polygon on the map represents a single zone area with a burden of anemia and severe anemia. High (red color) means high rates (hot spot) of anemia and severe anemia. Low (green color) shows a low rate (cold spot) of severe anemia and undefined (black color) indicates lakes and zones (did not sample taken in this area). To conduct this analysis use GeoDa version 1.14, which is available at: https://geodacenter.github.io/download_windows.html.

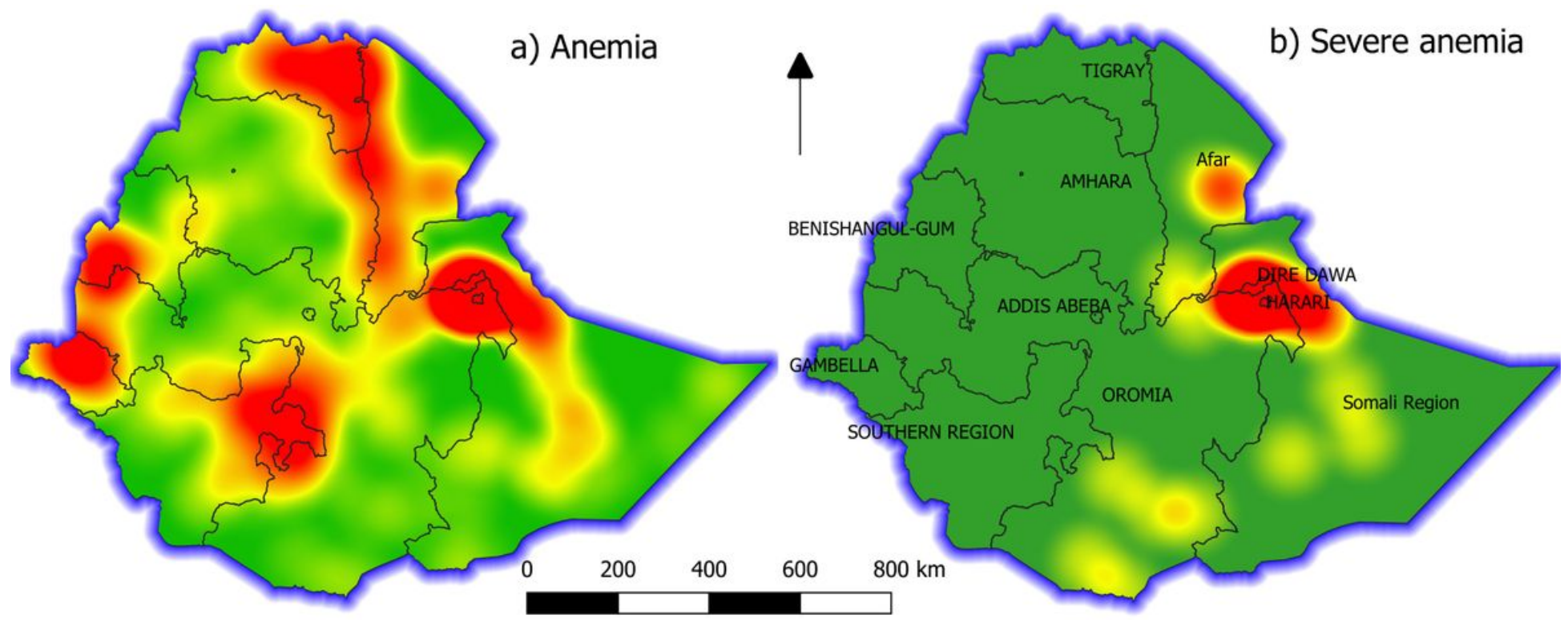

\section{Figure 5}

Heat map of anemia and severe anemia under-5 children in Ethiopia, 2016 In Figure 5. The red color indicates denser concentration of anemia in the area. Yellow color shows next high concentration or density of case. The green shows based on area the case as did not accumulate specific area, it is scattered throughout the area. The right panel of the map is identified anemia density and the left side of the panel severe anemia density. Or go to 
from red to green through yellow the concentration (density) is increased compared to a specific area (not based on people). To carry out this analysis used QGIS 3.4.3, which available at https://www.npackd.org/p/qgis/3.4.3.

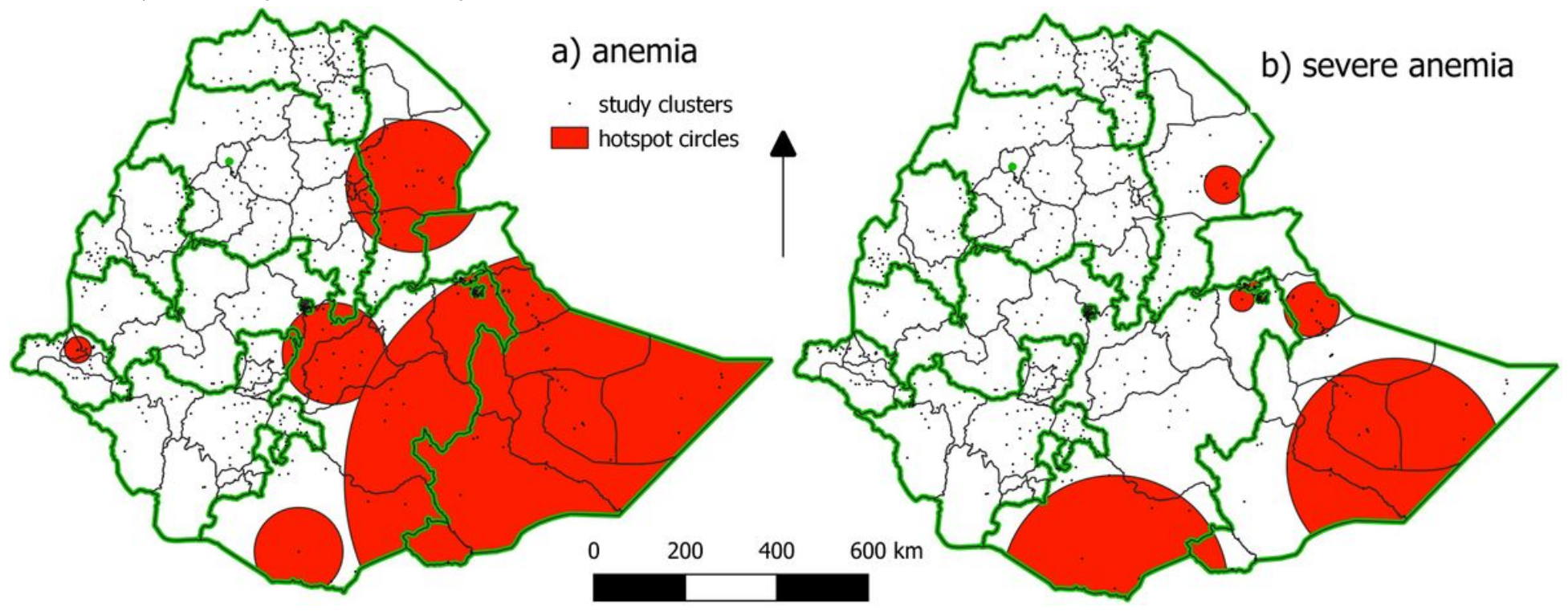

Figure 6

Anemia and severe anemia hotspot clusters identified using SaTScan spatial analysis tool, in Ethiopia 2016. The red color circle in both panels indicates hotspot windows. And the dot indicates enumeration cluster. To conduct this analysis use GeoDa version 1.14, which is available at: SaTScan v9.6, which available at https://www.satscan.org/download.html and QGIS 3.4.3.

\section{Supplementary Files}

This is a list of supplementary files associated with this preprint. Click to download.

- Additionalfile1.xlsx 